\title{
Mitteilungen UNION
}

\section{Neues Vorstandsmitglied Dr. Anita Meyer Hitz}

Der Vorstand der UNION freut sich, Dr. Anita Meyer Hitz (Abb. 1) als neues Vorstandsmitglied zu begrüssen. Dr. Anita Meyer Hitz vertritt neben Dr. Brigitte AusfeldHafter die Assoziation Schweizerischer Ärztegesellschaften für Akupunktur und Chinesische Medizin (ASA) im Vorstand der UNION. Die Wahl erfolgt an der nächsten Delegiertenversammlung der UNION.

\section{Vorstellung Dr. Anita Meyer Hitz}

«Nach dem Staatsexamen habe ich mich zur Fachärztin Innere Medizin FMH ausgebildet. Ich bekam 3 Kinder und folgte damit auch einer typischen Frauenkarriere der damaligen Zeit. Während der ersten 6 Jahre als Familienfrau und Mutter habe ich mich mit Praxisvertretungen, AIDSSprechstunden und später einem kleinen Pensum als Ärztin in einer Landarztpraxis beruflich à jour gehalten.

Immer schon habe ich mich für das Zusammenspiel von Seele und Körper interessiert. Mein vierjähriges Studium an der International Free University of Maastricht (IFU) hat mir mit der stark philosophisch-daoistisch geprägten Ausbildung in der Chinesischen Medizin ein neues Weltverständnis eröffnet. Vor 18 Jahren habe ich meine eigene Praxis für Akupunktur in Frauenfeld eröffnet. Eine zweijährige Ausbildung bei der AgTCM in Zürich brachte mir das ergänzende Wissen in der Arzneitherapie, sodass ich heute meine Praxis mit Schwerpunkt Akupunktur und wenn nötig unterstützend mit chinesischer Arzneitherapie führe.

Ich bin überzeugt, dass in der Medizin neben dem Körper auch die Seele ihre Beachtung finden muss - und zunehmend auch der lebensphilosophische Aspekt des Menschseins. Vor 2 Jahren habe ich deshalb ein Studium mit einem «CAS Medizin und Philosophie» an der Univer-

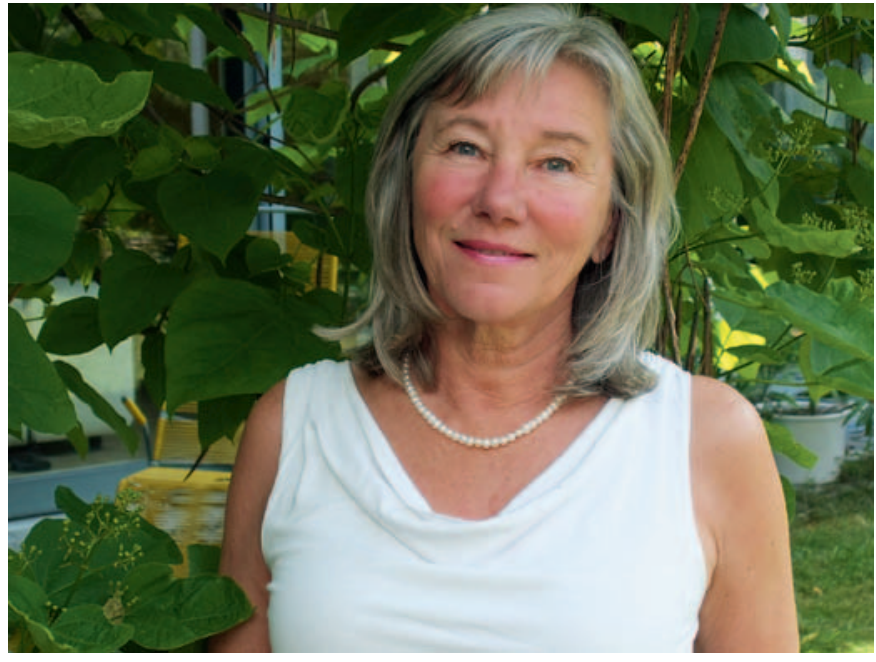

Abb. 1. Dr. Anita Meyer Hitz.

sität Luzern abgeschlossen. An internationalen Kongressen habe ich mich zudem ständig in Daoismus und Buddhismus in der Medizin weitergebildet. Die philosophischen Grundlagen unseres ärztlichen Tuns sind mir ein grosses Anliegen.

Mit dem Grösserwerden der Kinder wollte ich mit meiner Mitarbeit in standespolitischen Gremien etwas zurückgeben in die Gesellschaft, von der ich so viel profitieren konnte. Ich bin daher seit Langem Vorstandsmitglied in der SACAM (Schweizerische Ärztegesellschaft für Akupunktur, chinesische Medizin und Aurikulomedizin) und verantwortlich für die Öffentlichkeitsarbeit. Zudem bin ich seit Beginn im wissenschaftlichen Komitee des ASA TCM-Kongresses; vor 2 Jahren habe ich die Co-Leitung als Präsidentin übernommen. Im Kongress stehe ich ein für ein vielfältiges Programm, persönlich engagiere ich mich für eine Vertretung von Philosophie, Klassik und Geschichte der TCM.

\section{Agenda UNION}

Vorstandssitzung UNION

Vorstandssitzung UNION Vorstandssitzung UNION Vorstandssitzung UNION Vorstandssitzung UNION
Donnerstag, 30. Oktober 2014 (Nachmittag)

Donnerstag, 11. Dezember 2014 (Nachmittag)

Donnerstag, 12. Februar 2015 (Nachmittag)

Donnerstag, 23. April 2015 (Vormittag)

Donnerstag, 23. April 2015 (Nachmittag) 
Der ASA-Kongress, der dieses Jahr anfangs Dezember zum 8. Mal in Solothurn durchgeführt wird, ist eine wunderbare Plattform, die unterschiedlichen Facetten und Richtungen der Chinesischen Medizin, von der Klassik des Daoismus bis zur modernen Lasertherapie, aufzuzeigen. Die Akzeptanz der unterschiedlichsten Richtungen in der Chinesischen Medizin, das Interesse für moderne Strömungen ebenso wie für die Tradition - diesen Reichtum am ASA-Kongress aufrecht zu erhalten, ist mir sehr wichtig.

Als Präsidentin der ASA - ich habe das Amt diesen Frühling übernommen - möchte ich die Breite der Chinesischen Medizin erhalten und fördern, auch und gerade mit den verschiedenen Schulen, Denkrichtungen und Traditionen in der Deutschschweiz und der Romandie. Es sind weit mehr als nur verschiedene Sprachen, die uns trennen. Umso wichtiger ist der gegenseitige Respekt, das Interesse für- und aneinander!

Als Vertreterin der ASA in der UNION möchte ich die Kommunikation zwischen der UNION als Dachorganisation und der Basis verbessern. Ich möchte Brücken bauen auf dem Boden eines konstruktiven Miteinanders der vier komplementärmedizinischen Richtungen, Brücken auch zwischen der ASA und deren drei Basis-Organisationen SACAM (Schweizerische Ärztegesellschaft für Akupunktur, Chinesische Medizin und Aurikulomedizin), AGMAR (Association Genevoise des Médecins Acupuncteurs) und ATMA (Akademie für Taoistische Medizin und Akupunktur) sowie der UNION.

Ich bin überzeugt, dass wir nur im Miteinander stark sein können. Die ASA ist von den Mitgliederzahlen her die grösste Gesellschaft in der UNION. Die Akupunktur (TCM) ist seit Längerem breit bekannt und akzeptiert in der Bevölkerung. Trotzdem ist es mir ein Anliegen, die gesamte Palette der Komplementärmedizin positiv in der medizinischen Landschaft zu verankern. Durch den Einsatz in der UNION für das breite Gebiet der Komplementärmedizin stärken wir auch die ärztliche Akupunktur und die TCM.

Als Ärztin ist es mir wichtig, dass die Komplementärmedizin - auch die TCM - in Zukunft einen starken Platz in der ärztlichen Ausbildung und in der ärztlichen Berufsausübung einnimmt.»

Dr. Anita Meyer Hitz

\section{Internationale Phytotherapie-Tagung 2014}

Vom 18. bis 21. Juni 2014 fand die internationale Phytotherapie-Tagung 2014 in Winterthur mit über 500 Teilnehmenden statt. An den vier Kongresstagen standen Firmenbesuche, Vorträge (unter anderem Phytotherapie in der Geriatrie und Gerontologie) sowie Exkursionen im Fokus. Erstmals diskutierten die drei Vertreter der Zulassungsbehörden aus Deutschland, Österreich und der Schweiz an einem Tisch. Es besteht die Hoffnung, dass die Zulassungsentscheide gegenseitig übernommen werden könnten. Weitere Informationen zur Tagung finden Sie unter $h t t p: / /$ phytotherapie2014.smgp.ch.

\section{Verbleib der ärztlichen Komplementärmedizin in der Grundversicherung}

Der Präsident der UNION, Dr. Hansueli Albonico, blickt zurück auf 15 Jahre WZW-Kontroverse zum Verbleib der ärztlichen Komplementärmedizin in der sozialen Grundversicherung der Schweiz. Lesen Sie mehr dazu auf Seite 264 in dieser Ausgabe. 